\title{
Transport in GaAs/AlAs/GaAs [111] Tunnel Junctions
}

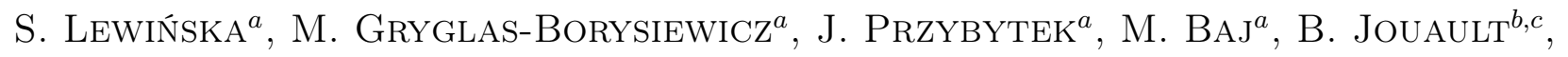 \\ U. Gennser ${ }^{d}$ And A. Ouerghi ${ }^{d}$ \\ ${ }^{a}$ Institute of Experimental Physics, Faculty of Physics, University of Warsaw, Hoża 69, 00-681 Warsaw, Poland \\ ${ }^{b}$ Groupe d'Etude des Semiconducteurs, Université Montpellier 2, Montpellier, France \\ ${ }^{c}$ CNRS, UMR 5650, cc074, pl. Eugène Bataillon, 34095 Montpellier Cedex 5, France \\ ${ }^{d}$ Laboratoire de Photonique et Nanostructures, CNRS, Marcoussis, France
}

\begin{abstract}
Resonant tunneling in single-barrier GaAs/AlAs/GaAs junctions grown in [111] direction was studied for samples with different concentration of silicon $\delta$-doping in AlAs. In the $I(V)$ characteristics, measured at $4 \mathrm{~K}$, two kinds of peaks were observed: related to resonant tunneling via donors states in the barrier, and through X-minimum quantum well subbands. The results are compared to those previously obtained for analogous samples grown along [001] direction. The investigations reveal different symmetry of donor states in both cases.
\end{abstract}

PACS: 73.61.-r, 71.20.Nr, 73.23.Hk, 73.63.Hs, 72.10.Di, 85.30.Mn, 73.40.Gk

\section{Introduction}

GaAs/AlAs/GaAs junctions with $\delta$-doping in the barrier are very interesting examples of tunneling devices: AlAs is a barrier for the electrons from $\Gamma$ point in the Brillouin zone and a quantum well for electrons from $X$ point. Under bias, energies of electrons in GaAs (emitter) can be aligned with the energies of the quantum well (QW) states in the barrier, which leads to resonant tunneling effect. If, in addition, the barrier is doped, also the donor states can participate in the resonant tunneling. This is especially interesting, as a discrete level of a single impurity can be used as a local probe of the tunneling electron gas $[1,2]$. However, if the structure is grown along [001] direction, due to strain and anisotropy of $\mathrm{X}$ minimum, the donor state is split into two components separated by $\approx 20 \mathrm{meV}$ [3]. Therefore even for one impurity, there were two probes instead of one, which was unfavorable for the studies. However, for structures grown along [111] direction no splitting was expected as the three $X$-minimum valleys should be equivalent. In the paper we present preliminary results of the studies of the [111] samples and compare them with [001] results $[4,5]$.

\section{Samples and measurement technique}

Samples were grown by MBE on the [111] GaAs $n^{+}$ substrate. They consisted of: $510 \mathrm{~nm}$ of $n^{+}$Si-doped $\left(4 \times 10^{18} \mathrm{~cm}^{-3}\right)$ GaAs, $100 \mathrm{~nm}$ of GaAs $\left(2 \times 10^{16} \mathrm{~cm}^{-3}\right)$, $100 \mathrm{~nm}$ of undoped GaAs, $10.2 \mathrm{~nm}$ of AlAs barrier, after which an inverse sequence of layer was grown to maintain the symmetry in respect of the barrier. Additionally, the barrier was $\delta$-doped in the center with silicon. Three types of samples were investigated: first with doping on the level of $10 \times 10^{9} \mathrm{~cm}^{-2}$, second: $5 \times 10^{9} \mathrm{~cm}^{-2}$; third: with no intentional doping (a reference sample). Circular junctions with diameters of 125, 250 and $500 \mu \mathrm{m}$ were prepared by optical lithography. Two kinds of transport measurements were performed: (1) current-voltage characteristics, where a DC bias was applied between the top contact and bottom electrode and tunneling current was measured, and (2) current noise spectral density, where the fluctuations of tunneling current for a fixed bias were measured by means of cross-correlation technique $[6,7]$. All the measurements were made at $4 \mathrm{~K}$.

\section{Results and discussion}

The typical current-voltage characteristics of the three samples studied are shown in Fig. 1. There are several broad "bumps" observed in the $I(V)$ curves, in both directions of polarizations, which are attributed to the resonant tunneling. First bump, at about $0.9 \mathrm{~V}$, sensitive to the doping level (see the inset), indicates the resonance through the donor states in the barrier. The fact that it is so broad can be understood taking into account huge number of donors $(\approx 3$ millions) present within the junction. The peaks observed at higher bias $(U \approx 1.5 \mathrm{~V}$ and $U \approx 2 \mathrm{~V}$ ) are due to tunneling via $X$-minimum quantum well subbands, which are higher in the energy than the donor states, and thus a higher bias is needed to align them with the electrons in the emitter. For the sample with the highest donor concentration a negative differential resistance is observed. Moreover, for all the samples a certain asymmetry of the $I(V)$ characteristics is seen. Most probably, it is related to the built-in piezoelectric field present in the structure [8]. 


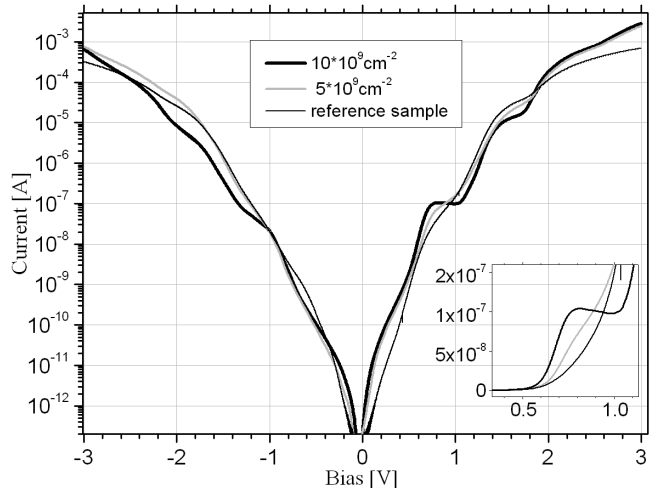

Fig. 1. $I(V)$ characteristics for [111] samples. Black line indicates the highest doping, grey - middle doping and light grey - the reference sample. The inset shows the zoom of negative differential resistance for heavily doped sample.

Clear evidence that the "bumps" in Fig. 1 are related to resonant tunneling (so that the new transport mechanism appears) comes from the noise measurements. Figure 2 shows the current noise spectrum, measured for the sample with the highest doping. For low bias $(U=0.4 \mathrm{~V})$ only the shot noise with a flat frequency dependence is observed. However, at biases higher than $0.6 \mathrm{~V}$, the generation-recombination noise appears in the low frequency part of the spectrum, which indicates that some electron traps (e.g. donor states) are active in the tunneling process $[6,7]$. More quantitative results come from the analysis proposed in [9] (subtracting the shot noise value and then dividing by a square of current for a given bias), which visualize the relative modulation noise spectral density. The result of such analysis is shown in Fig. 3. One can see that for certain values of bias the modulation noise is strongly increased (at about $0.5 \mathrm{~V}, 1.25 \mathrm{~V}$, $1.75 \mathrm{~V})$. Interestingly, at these biases sharp current increases are observed (see Fig. 1). The observed character of modulation noise reveals the quantum character of the current flow - if the channels for electron transport are closed or fully open, there is no noise related to the current. On the contrary, for the partially open/closed channels, the noise has maximum (behaviour similar as for ballistic transport in quantum point contacts - see e.g. [9]).

The result of transport experiments for [111] samples are compared with [100] samples $[4,5]$. Figure 4 shows the current densities obtained from the $I(V)$ characteristics for the two sets of samples. The concentration of $\delta$-doping in these two structures is comparable and equals $3 \times 10^{9} \mathrm{~cm}^{-2}$ for [001] and $5 \times 10^{9} \mathrm{~cm}^{-2}$ for [111]. The curves are quite similar with characteristic bumps due to resonant tunneling. For $U \leq 0.5 \mathrm{~V}$ the values of current densities are very much alike for both samples whereas at higher bias the current density for [001] is systematically higher. The positions of the current maxima are roughly the same for both samples. However, a detailed

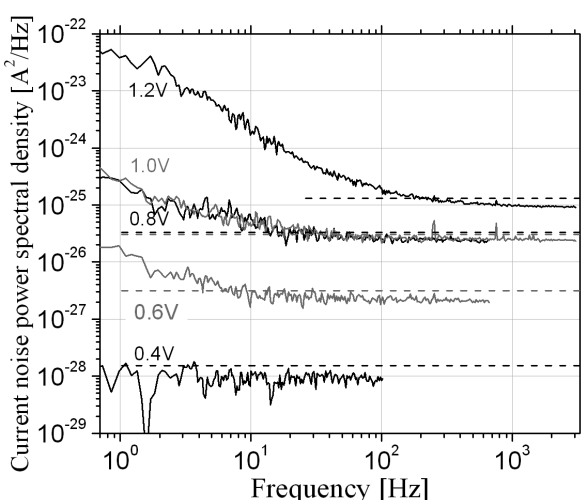

Fig. 2. Current noise power spectral density $\left(S_{I}\right)$ for the heavily doped [111] sample for several values of the bias applied, as indicated on the left. Horizontal dashed lines indicate the values of the full shot noise of uncorrelated electrons. For a given bias its value is calculated after the Schottky formula: $S_{I}=2 e I$. The fact that the measured shot noise (higher-frequency flat part of the spectrum) is lower than full shot noise indicates that the electrons passing through a barrier tend to correlate.

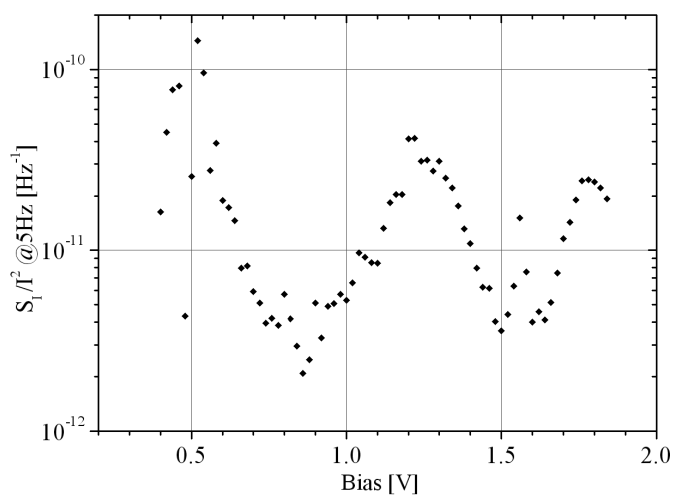

Fig. 3. Relative modulation noise spectral density versus bias voltage measured at $5 \mathrm{~Hz}$ for the heavily doped [111] sample.

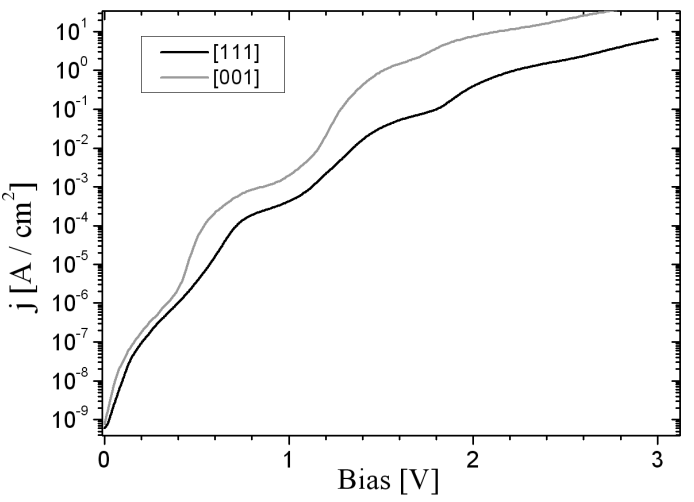

Fig. 4. Current density as a function of bias for [001] and [111] samples. 


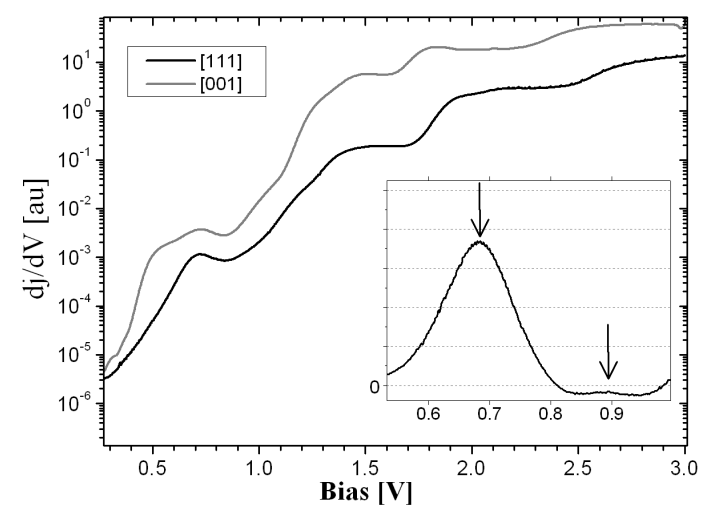

Fig. 5. First derivative of current density for [001] and [111] samples. The inset shows the result for the sample with the highest doping (vertical scale is linear).

structure of the maxima is different. This is well seen in Fig. 5, where a derivate of current density was calculated. The first bump, starting at $\approx 0.6 \mathrm{~V}$, attributed to the tunneling via donor states, is composed of two parts for [001] sample and of one part for [111]. The latter fact is indeed expected for a shallow donor below $X$ minimum, as the three minima are equivalent. However, surprisingly, for a higher concentration of impurities one sees two peaks (indicated by arrows in the inset to Fig. 5). It is not clear at the moment whether the second peak comes from phonon replica or decreased symmetry of the donor environment.

\section{Conclusions}

Single barrier structures with impurities embedded in the barrier, grown in [111] direction were studied. The $I(V)$ characteristics and noise measurements allowed to identify the resonant tunneling, through donor states and through $X$-minimum quantum well subbands. For moderate concentrations of $\delta$-doping donor levels are orientationally degenerate, in contrast with previous observations on [001] samples. However, when the doping is increased the degeneracy of donor level is lifted. The origin of this effect is unknown.

\section{Acknowledgments}

Work has been partially supported by the Polish Ministry of Science and Higher Education project No. N N202 192534.

\section{References}

[1] M. Gryglas, M. Baj, B. Chenaud, B. Jouault, A. Cavanna, G. Faini, Phys. Rev. B 69, 165302 (2004).

[2] B. Jouault, M. Gryglas, G. Faini, U. Gennser, A. Cavanna, M. Baj, D.K. Maude, Phys. Rev. B 73, 155415 (2006).

[3] I.E. Itskevich, L. Eaves, P.C. Main, M. Henini, G. Hill, Phys. Rev. B 57, 7214 (1998).

[4] M. Gryglas, J. Przybytek, M. Baj, M. Henini, L. Eaves, Acta Phys. Pol. A 100, 403 (2001).

[5] Yu.N. Khanin, K.S. Novoselov, E.E. Vdovin, Semiconductors 35, 206 (2001).

[6] J. Przybytek, M. Baj, Acta Phys. Pol. A 112, 221 (2007).

[7] J. Przybytek, M. Baj, Am. Inst. Phys. Conf. Proc. 1129, 333 (2009).

[8] J.A. Majewski, P. Vogl, P. Lugli, in: Proc. 25th Int. Conf. Semiconductors, Osaka, 2000, Springer, Heidelberg 2001.

[9] Sh. Kogan, Electronic Noise and Fluctuations in Solids, Cambridge University Press, 1996. 